\title{
DETERMINATION OF PARAMETERS IN TELEGRAPH EQUATION
}

\section{A.I. KOZHANOV, R.R. SAFIULLOVA}

\begin{abstract}
We study the solvability of the inverse problems on finding a solution $u(x, t)$ and an unknown coefficient $c$ for a telegraph equation

$$
u_{t t}-\Delta u+c u=f(x, t) .
$$

We prove the theorems on the existence of the regular solutions. The feature of the problems is a presence of new overdetermination conditions for the considered class of equations.
\end{abstract}

Keywords: telegraph equation, unknown coefficient, inverse problems, special type integral overdetermination, regular solutions, existence.

Mathematics Subject Classification: 35R30, 35L20

\section{INTRODUCTION}

Mathematical modelling of oscillating processes (propagation of electromagnetic waves [1], [2], of acoustic waves [3], [4], etc.) leads one to studying the solvability of various boundary value problems and the properties of the solutions of the equation

$$
u_{t t}-a^{2} \Delta u+c u=f(x, t)
$$

called a telegraph equation. Here the coefficients $a$ and $c$ are positive and are determined by the properties of a media. In the case of a media with apriori unknown properties these coefficients, both or one of them, are unknown and determination of them allow one to analyze a physical process employing the telegraph equation with all known data.

In the present work we study the case of the unknown coefficient $c$, for simplicity we assume that $a=1$.

The problems in which one need to determine both a solution and a coefficient or coefficients of a differential equation or the right hand side of the equation are called inverse problem in mathematics and mathematical modelling. As a rule, in such problems, apart of boundary and initial conditions specific for some class of differential equations, other additional conditions are imposed, which are usually called overdetermination conditions. The theory of inverse problems for differential equations is an actively developing direction in the modern mathematics. In particular, the solvability of the inverse problems in various formulations with various overdetermination conditions for hyperbolic equations was studied in in many works, see monographs [4]- 9], papers [10]-[23] and others. At the same time we note, that the inverse problems for the telegraph equation with overdetermination conditions in the present paper were not studied before.

A.I. Kozhanov, R.R. Safiullova, Determination of parameters in telegraph equation.

(c) Kozhanov A.I., Safiullova R.R. 2017.

The work is supported by Russian Foundation for Basic Research (project no. 15-01-06582).

Submitted February 14, 2016. 


\section{Formulation OF THE PROBlems}

Let $\Omega$ be a bounded domain in the space $\mathbb{R}^{n}$ with a smooth (infinitely differentiable) boundary $\Gamma, Q$ be a cylinder $\Omega \times(0, T)$ of a finite height $T, S=\Gamma \times(0, T), f(x, t), u_{0}(x), u_{1}(x)$ be given functions defined as $x \in \bar{\Omega}$ and as $t \in[0, T], A$ be a given positive number.

Inverse problem I: find a function $u(x, t)$ and a number $c$ such that in the cylinder $Q$ the equation

$$
u_{t t}-\Delta u+c u=f(x, t)
$$

is satisfied and at that, for the function $u(x, t)$ the conditions

$$
\begin{aligned}
& u(x, 0)=u_{0}(x), \quad u_{t}(x, 0)=u_{1}(x), \quad x \in \Omega, \\
& \left.u(x, t)\right|_{S}=0, \\
& \int_{\Omega} u^{2}(x, T) d x=A .
\end{aligned}
$$

Inverse problem II: find a function $u(x, t)$ and a number $c$ such that in the cylinder $Q$ equation (1) is satisfied and at that, for the function $u(x, t)$ conditions (2) and (4) hold as well as the condition

$$
\left.\frac{\partial u(x, t)}{\partial \nu_{x}}\right|_{S}=0
$$

$\nu_{x}=\left(\nu_{1}, \ldots, \nu_{n}\right)$ is the inner normal for $\Gamma$ at a point $x$.

In Inverse problems I-II conditions (2) and (3), (2) and (5) are the conditions for the Dirichlet or Neumann initial boundary value problems for a second order hyperbolic equation, while condition (4) is an overdetermination condition. The inverse problems for the hyperbolic equations with such overdetermination condition was not studies before. At the same time, we note that in works [24]-[26], inverse problems of close formulation for hyperbolic equations were studied, but the methods of these works of are not applied directly to Inverse problems I, II.

And one more remark before the substantial part of the work. The solvability of Inverse problems I and II can be established formally by means of the Fourier method. Namely, regarding the coefficient $c$ as known, representing a solution to the Dirichlet and Neumann initial boundary value problem for equation (1) by a Fourier series and employing then overdetermination condition (4), we obtain an algebraic condition for the coefficient $c$. But it is obvious that this equation has a rather complicated structure and it is not easy to give relatively simple solvability conditions of this equation. The method we propose below gives conditions, which can be checked easily in particular problems and this method can be also applied in situations more general than considered here.

\section{Solvability of InVERse Problem I}

The solvability of Inverse problem I is based on studying the solvability of the Dirichlet initial boundary value problem for some auxiliary nonlinear integral differential ("loaded" [27], [28]) equation. In its turn, the proof of solvability of the direct Dirichlet initial boundary value problem for the auxiliary equation is based on the cut-off functions method, regularization method and the fixed point method.

For the sake of brevity we denote

$$
A_{0}=\int_{\Omega} u_{0}^{2}(x) d x
$$


and in what follows we assume the condition

$$
A_{0}<A \text {. }
$$

We introduce extra notations:

$$
B_{0}=\sum_{i=1}^{n} \int_{\Omega} u_{0 x_{i}}^{2}(x) d x, \quad B_{1}=\int_{\Omega} u_{1}^{2}(x) d x, \quad b_{0}=\frac{B_{0}+B_{1}}{A-A_{0}}, \quad b_{1}=\frac{1}{A-A_{0}}, \quad \beta=\frac{b_{0}}{b_{1}} .
$$

Given a fixed positive number $N$, we define a cut-off function $G_{N}(\xi)$ :

$$
G_{N}(\xi)= \begin{cases}\xi, & \text { as } \quad 0 \leqslant \xi \leqslant N \\ N, & \text { as } \quad \xi>N \\ 0, & \text { as } \quad \xi<0\end{cases}
$$

Given a function $v(x, t)$ in the space $W_{2}^{2}(Q)$, we denote by $\Phi(v)$ the number

$$
\Phi(v)=\int_{\Omega} v_{t}^{2}(x, T) d x+\sum_{i=1}^{n} \int_{\Omega} v_{x_{i}}^{2}(x, T) d x-2 \int_{Q} f v_{t} d x d t .
$$

We consider the problem: find a function $u(x, t)$ solving the equation

$$
u_{t t}-\Delta u+\left[b_{0}-b_{1} G_{\beta}(\Phi(u))\right] u=f(x, t)
$$

in the cylinder $Q$ and obeying conditions (2) and (3).

Exactly problem (7), (2), (3) is auxiliary. The solvability of this problem will allow us to establish the solvability of Inverse problem I.

Theorem 1. Let the functions $f(x, t), u_{0}(x)$ and $u_{1}(x)$ be such that

$$
f(x, t) \in L_{2}(Q), \quad f_{t}(x, t) \in L_{2}(Q), \quad u_{0}(x) \in W_{2}^{2}(\Omega) \cap \stackrel{\circ}{W}_{2}^{1}(\Omega), \quad u_{1}(x) \in \stackrel{\circ}{W}_{2}^{1}(\Omega) .
$$

Moreover, assume that condition (6) is satisfied. Then boundary value problem (7), (2), (3) has a solution $u(x, t)$ such that $u(x, t) \in L_{\infty}\left(0, T ; W_{2}^{2}(\Omega) \cap \stackrel{\circ}{W}_{2}^{1}(\Omega)\right), u_{t}(x, t) \in L_{\infty}\left(0, T ; \stackrel{\circ}{W}_{2}^{1}(\Omega)\right)$, $u_{t t}(x, t) \in L_{\infty}\left(0, T ; L_{2}(\Omega)\right)$.

Proof. We employ the regularization method and the fixed point method .

Let $\varepsilon$ be a positive number. We consider the following problem: find a function $u(x, t)$ solving the equation

$$
u_{t t}-\Delta u+\left[b_{0}-b_{1} G_{\beta}(\Phi(u))\right] u-\varepsilon \Delta u_{t}=f(x, t)
$$

in the cylinder $Q$ and obeying conditions (2) and (3).

We introduce the linear space $V$ :

$$
\begin{aligned}
V=\left\{v(x, t): v(x, t) \in L_{\infty}\left(0, T ; W_{2}^{2}(\Omega) \cap \stackrel{\circ}{W}_{2}^{1}(\Omega)\right), v_{t}(x, t) \in L_{\infty}\left(0, T ; \stackrel{\circ}{W}_{2}^{1}(\Omega)\right),\right. \\
\left.v_{t t}(x, t) \in L_{2}(Q), \Delta v_{t}(x, t) \in L_{2}(Q)\right\} .
\end{aligned}
$$

We equip the space $V$ by the norm

$$
\|v\|_{V}=\left[\|v\|_{L_{\infty}\left(0, T ; W_{2}^{2}(\Omega) \cap \stackrel{\circ}{2}_{2}^{1}(\Omega)\right)}^{2}+\left\|v_{t}\right\|_{L_{\infty}\left(0, T ; \dot{W}_{2}^{1}(\Omega)\right)}^{2}+\left\|v_{t t}\right\|_{L_{2}(Q)}^{2}+\left\|\Delta v_{t}\right\|_{L_{2}(Q)}^{2}\right]^{\frac{1}{2}} .
$$

The space $V$ equipped with this norm is obviously a Banach space.

By using the fixed point method, let us show that boundary value problem $\left(7_{\varepsilon}\right),(2),(3)$ is solvable in the space $V$ for a fixed $\varepsilon$ and if the function $f(x, t)$ is an element of the space $L_{2}(Q)$.

Let $w(x, t)$ be a function in the space $V$. We consider the problem: find a function $u(x, t)$ solving the equation

$$
u_{t t}-\Delta u+\left[b_{0}-b_{1} G_{\beta}(\Phi(w))\right] u-\varepsilon \Delta u_{t}=f(x, t)
$$


in the cylinder $Q$ and obeying conditions (2) and (3).

Boundary value problem $\left(7_{\varepsilon, w}\right),(2),(3)$ is the Dirichlet initial boundary value problem for a linear pseudo-hyperbolic equation with constant coefficients and its solvability in the space $V$ for a function $f(x, t)$ in the space $L_{2}(Q)$ is known, see [29], [30]. Therefore, this problem generates the operator $\Re$ mapping the space $V$ into itself: $\Re(w)=u$. Let us show that the operator $\Re$ has fixed points in the space $V$.

We consider the identity

$$
\int_{0}^{t} \int_{\Omega}\left\{u_{\tau \tau}-\Delta u+\left[b_{0}-b_{1} G_{\beta}(\Phi(w))\right] u-\varepsilon \Delta u_{\tau}\right\} u_{\tau} d x d \tau=\int_{0}^{t} \int_{\Omega} f u_{\tau} d x d \tau .
$$

Integrating by parts, it is easy to pass from this identity to the following one:

$$
\begin{aligned}
\frac{1}{2} \int_{\Omega} u_{t}^{2}(x, t) d x & +\frac{1}{2} \sum_{i=1}^{n} \int_{\Omega} u_{x_{i}}^{2}(x, t) d x+\varepsilon \sum_{i=1}^{n} \int_{0}^{t} \int_{\Omega} u_{x_{i} \tau}^{2} d x d \tau \\
& +\frac{1}{2}\left[b_{0}-b_{1} G_{\beta}(\Phi(w))\right] \int_{\Omega} u^{2}(x, t) d x \\
= & \frac{1}{2}\left(B_{0}+B_{1}+b_{0} A_{0}\right)-\frac{1}{2} b_{1} A_{0} G_{\beta}(\Phi(w))+\int_{0}^{t} \int_{\Omega} f u_{\tau} d x d \tau
\end{aligned}
$$

We observe that the inequalities

$$
0 \leqslant b_{0}-b_{1} G_{\beta}(\Phi(w)) \leqslant b_{0}
$$

hold true. We denote

$$
N_{0}=B_{0}+B_{1}+b_{0} A_{0}+\int_{Q} f^{2} d x d t
$$

Employing inequalities (9) and applying Grönwall lemma, we obtain by (8) the estimates

$$
\begin{aligned}
& \int_{0}^{t} \int_{\Omega} u_{\tau}^{2} d x d \tau \leqslant N_{0}\left(e^{t}-1\right), \\
& \int_{\Omega} u_{t}^{2}(x, t) d x+\sum_{i=1}^{n} \int_{\Omega} u_{x_{i}}^{2}(x, t) d x+\varepsilon \sum_{i=1}^{n} \int_{0}^{t} \int_{\Omega} u_{x_{i} \tau}^{2} d x d \tau \leqslant N_{0} e^{t},
\end{aligned}
$$

which are valid for all $t$ in the segment $[0, T]$.

At the next step we consider the identity

$$
\int_{0}^{t} \int_{\Omega}\left\{u_{\tau \tau}-\Delta u+\left[b_{0}-b_{1} G_{\beta}(\Phi(w))\right] u-\varepsilon \Delta u_{\tau}\right\}\left(u_{\tau \tau}-\Delta u_{\tau}\right) d x d \tau=\int_{0}^{t} \int_{\Omega} f\left(u_{\tau \tau}-\Delta u_{\tau}\right) d x d \tau .
$$

We integrate by parts, employ estimates (10) and (11) and apply Young inequality to obtain that for all possible solutions $u(x, t)$ of problem $\left(7_{\varepsilon, w}\right),(2),(3)$ the estimate

$$
\|u\|_{V} \leqslant R_{0}
$$

holds true, where the number $R_{0}$ is determined just by the functions $f(x, t), u_{0}(x), u_{1}(x)$ and the numbers $\varepsilon, A, T$. 
Estimate (13) means that the operator $\Re$ maps a closed ball of radius $R_{0}$ in the space $V$ into itself.

Let us show that the operator $\Re$ is continuous on the space $V$. Let $\left\{w_{m}(x, t)\right\}_{m=1}^{\infty}$ be a sequence of functions in the space $V$ converging in this space to the function $w_{0}(x, t)$, $\left\{v_{m}(x, t)\right\}_{m=1}^{\infty}$ be a sequence of images of the functions $w_{m}(x, t)$ under the action of the operator $\Re, v_{0}(x, t)$ be the image of the function $w_{0}(x, t)$ under the action of the operator $\Re$. We denote $\bar{w}_{m}(x, t)=w_{m}(x, t)-w_{0}(x, t), \bar{v}_{m}(x, t)=v_{m}(x, t)-v_{0}(x, t)$. The identities

$$
\begin{aligned}
& \bar{v}_{m t t}-\Delta \bar{v}_{m}+\left[b_{0}-b_{1} G_{\beta}\left(\Phi\left(w_{m}\right)\right)\right] \bar{v}_{m}-\varepsilon \Delta \bar{v}_{m t}=b_{1}\left[G_{\beta}\left(\Phi\left(w_{m}\right)\right)-G_{\beta}\left(\Phi\left(w_{0}\right)\right)\right] v_{0}, \quad(x, t) \in Q \\
& \bar{v}_{m}(x, 0)=\bar{v}_{m t}(x, 0)=0, \quad x \in \Omega,\left.\quad \bar{v}_{m}(x, t)\right|_{S}=0
\end{aligned}
$$

hold true. These identities imply the estimate

$$
\begin{aligned}
\int_{\Omega} \bar{v}_{m t}^{2}(x, t) d x & +\sum_{i=1}^{n} \int_{\Omega} \bar{v}_{m x_{i}}^{2}(x, t) d x+\varepsilon \sum_{i=1}^{n} \int_{0}^{t} \int_{\Omega} \bar{v}_{m x_{i} \tau}^{2} d x d \tau \\
& \leqslant \int_{0}^{t} \int_{\Omega} v_{m \tau}^{2} d x d \tau+b_{1}^{2}\left[G_{\beta}\left(\Phi\left(w_{m}\right)\right)-G_{\beta}\left(\Phi\left(w_{0}\right)\right)\right]^{2} \int_{Q} v_{0}^{2} d x d t .
\end{aligned}
$$

We observe that the function $G_{\beta}(\xi)$ satisfies the Lipschitz condition and the convergence $\Phi\left(w_{m}\right)-\Phi\left(w_{0}\right) \rightarrow 0$ holds true as $m \rightarrow \infty$. The latter is implied by the continuity of the norm and by the fact that the strong convergence implies the weak one. Taking into consideration these facts and applying Grönwall lemma, by (14) we obtain the convergence

$$
\left\|\bar{v}_{m}\right\|_{L_{\infty}\left(0, T ; W_{2}^{1}(\Omega)\right)} \rightarrow 0
$$

as $m \rightarrow \infty$.

We consider the identity

$$
\begin{gathered}
\left.\int_{0}^{t} \int_{\Omega} \bar{v}_{m \tau \tau}-\Delta \bar{v}_{m}+\left[b_{0}-b_{1} G_{\beta}\left(\Phi\left(w_{m}\right)\right)\right] \bar{v}_{m}-\varepsilon \Delta \bar{v}_{m \tau}\right\}\left(\bar{v}_{m \tau \tau}-\Delta \bar{v}_{m \tau}\right) d x d \tau \\
\quad=b_{1} \int_{0}^{t} \int_{\Omega}\left[G_{\beta}\left(\Phi\left(w_{m}\right)\right)-G_{\beta}\left(\Phi\left(w_{0}\right)\right)\right]\left(\bar{v}_{m \tau \tau}-\Delta \bar{v}_{m \tau}\right) d x d \tau .
\end{gathered}
$$

Integrating by parts and taking into consider the Lipschitz property of the function $G_{\beta}(\xi)$ once again as well as the established convergence, it is easy to show that this identity implies an apriori estimate for the family $\left\{\bar{v}_{m}(x, t)\right\}_{m=1}^{\infty}$ in the space $V$ and the convergence

$$
\left\|\bar{v}_{m}\right\|_{V} \rightarrow 0 \quad \text { as } \quad m \rightarrow \infty
$$

This convergence does mean that the operator $\Re$ is continuous in the space $V$.

Let us show that the operator $\Re$ is compact.

Let $\left\{w_{m}(x, t)\right\}_{m=1}^{\infty}$ is a bounded family in the space $V$. Since the families $\left\{w_{m t}(x, t)\right\}_{m=1}^{\infty}$, $\left\{w_{m x_{i}}(x, t)\right\}_{m=1}^{\infty}, i=1, \ldots, n$, are bounded in the space $W_{2}^{1}(Q)$ and since the embeddings $W_{2}^{1}(Q) \subset L_{2}(Q), W_{2}^{1}(Q) \subset L_{2}(\partial Q)$ are compact (see [31], [32]), there exits a subsequence 
$\left\{w_{m_{k}}(x, t)\right\}_{m=1}^{\infty}$ and a function $w_{0}(x, t)$ in the space $V$ such that, as $k \rightarrow \infty$, we have

$$
\begin{array}{lll}
w_{m_{k}}(x, t) \rightarrow w_{0}(x, t) & \text { weakly in } & W_{2}^{2}(Q), \\
\Delta w_{m_{k} t}(x, t) \rightarrow \Delta w_{0 t}(x, t) & \text { weakly in } L_{2}(Q), \\
w_{m_{k} t}(x, T) \rightarrow w_{0 t}(x, T) & \text { strongly in } L_{2}(\Omega), \\
w_{m_{k} x_{i}}(x, T) \rightarrow w_{0 x_{i}}(x, T) & \text { strongly in } L_{2}(\Omega) \text { for } i=1, \ldots, n .
\end{array}
$$

We again define the functions $v_{m}(x, t), v_{0}(x, t)$ as the images of the functions $w_{m}(x, t), w_{0}(x, t)$ under the action of the operator $\Re$. Reproducing the proof of the continuity of the operator $\Re$, we obtain that the convergence

$$
\left\|\bar{v}_{m_{k}}\right\|_{V} \rightarrow 0 \quad \text { as } \quad k \rightarrow \infty .
$$

holds true. In other words, each bounded sequence $\left\{w_{m}(x, t)\right\}_{m=1}^{\infty}$ of elements in the space $V$ contains a subsequence $\left\{w_{m_{k}}(x, t)\right\}_{k=1}^{\infty}$ such that the sequence $\left\{\Re\left(w_{m_{k}}\right)\right\}_{k=1}^{\infty}$ converges strongly in $V$. This means that the operator $\Re$ is compact.

Thus, the operator $\Re$ maps a closed ball of radius $R_{0}$ in the space $V$ into itself, is continuous and compact in the space $V$. By the Schauder theorem [33], the operator $\Re$ has at least one fixed point in this ball. For this fixed point, the equation $\left(7_{\varepsilon}\right)$ holds true and conditions (2) and (3) are satisfied. We denote the sought fixed point by $u^{\varepsilon}(x, t)$. Let us show that under additional assumption $f_{t}(x, t) \in L_{2}(Q)$, the family of the functions $\left\{u^{\varepsilon}(x, t)\right\}$ contains a sequence converging to a solution of boundary value problem (7), (2), (3).

We choose a sequence $\left\{\varepsilon_{m}\right\}_{m=1}^{\infty}$ of positive numbers such that $\varepsilon_{m} \rightarrow 0$ as $m \rightarrow \infty$.

Reproducing the proofs of estimates (10), (11) and (13) but integrating by parts w.r.t. the variable $\tau$ in the term $f \Delta u_{\tau}^{\varepsilon_{m}}$ in the right hand side of (12), it is easy to obtain the estimate

$$
\varepsilon_{m} \int_{Q}\left(\Delta u_{\tau}^{\varepsilon_{m}}\right)^{2} d x d \tau+\left\|\Delta u^{\varepsilon_{m}}\right\|_{L_{\infty}\left(0, T ; L_{2}(\Omega)\right)}^{2}+\left\|u_{t t}^{\varepsilon_{m}}\right\|_{L_{2}(Q)}^{2} \leqslant M_{1}
$$

with a constant $M_{1}$ independent of $\varepsilon$. Employing the compactness of the embeddings $W_{2}^{1}(Q) \subset L_{2}(Q), W_{2}^{1}(Q) \subset L_{2}(\partial Q)$, we obtain that there exists a sequence $\left\{m_{k}\right\}_{k=1}^{\infty}$ of natural numbers and a function $u(x, t)$ such that as $k \rightarrow \infty$ the convergences

$$
\begin{array}{ll}
\varepsilon_{m_{k}} \rightarrow 0, & \\
u^{\varepsilon_{m_{k}}}(x, t) \rightarrow u(x, t) & \text { weakly in } W_{2}^{2}(Q), \\
\varepsilon_{m_{k}} \Delta u_{t}^{\varepsilon_{m_{k}}}(x, t) \rightarrow 0 & \text { weakly in } L_{2}(Q), \\
u_{t}^{\varepsilon_{m_{k}}}(x, T) \rightarrow u_{t}(x, T) & \text { strongly in } L_{2}(\Omega), \\
u_{x_{i}}^{\varepsilon_{m_{k}}}(x, T) \rightarrow u_{x_{i}}(x, T) & \text { strongly in } L_{2}(\Omega) \text { for } i=1, \ldots, n, \\
\Phi\left(u^{\varepsilon_{m_{k}}}\right) \rightarrow \Phi(u) . &
\end{array}
$$

These convergences imply that the limiting function $u(x, t)$ solves equation $(7)$ and obeys conditions (2) and (3). The limiting function $u(x, t)$ belongs to $L_{\infty}\left(0, T ; W_{2}^{2}(\Omega) \cap W_{2}^{1}(\Omega)\right)$. This belonging and the belonging of the function $f(x, t)$ to the space $L_{\infty}\left(0, T ; L_{2}(\Omega)\right)$ imply that the function $u_{t t}(x, t)$ belongs to the same space. In other words, the limiting function $u(x, t)$ belongs to the sought class and in this way it is the required solution to boundary value problem $(7),(2),(3)$.

We proceed to studying the solvability of Inverse problem I.

The most simple case is when in Problem I we have $f(x, t) \equiv 0$. 
Theorem 2. Let the functions $f(x, t), u_{0}(x)$ and $u_{1}(x)$ be such that

$$
f(x, t) \equiv 0, \quad u_{0}(x) \in W_{2}^{2}(\Omega) \cap \stackrel{\circ}{W}_{2}^{1}(\Omega), \quad u_{1}(x) \in \stackrel{\circ}{W}_{2}^{1}(\Omega)
$$

and condition (6) be satisfied. Then Inverse problem $I$ has the solution $\{u(x, t), c\}$ such that $u(x, t) \in L_{\infty}\left(0, T ; W_{2}^{2}(\Omega) \cap \stackrel{\circ}{W}_{2}^{1}(\Omega)\right), u_{t}(x, t) \in L_{\infty}\left(0, T ; \stackrel{\circ}{W}_{2}^{1}(\Omega)\right), u_{t t}(x, t) \in L_{\infty}\left(0, T ; L_{2}(\Omega)\right)$, $c \geqslant 0$.

Proof. Under the assumptions of the theorem boundary value problem (7), (2), (3) has the solution $u(x, t)$ belonging to class mentioned in the theorem. We observe that in the case $f(x, t) \equiv 0$ we have $\Phi(u) \geqslant 0$. We multiply equation $(7)$ by the function $u_{t}(x, t)$ and integrate over the cylinder $Q$. We obtain the identity

$$
\begin{aligned}
\frac{1}{2} \int_{\Omega} u_{t}^{2}(x, T) d x & +\frac{1}{2} \sum_{i=1}^{n} \int_{\Omega} u_{x_{i}}^{2}(x, T) d x+\frac{b_{0}-b_{1} G_{\beta}(\Phi(u))}{2} \int_{\Omega} u^{2}(x, T) d x \\
& =\frac{1}{2}\left(B_{0}+B_{1}+b_{0} A_{0}\right)-\frac{b_{1} A_{0} G_{\beta}(\Phi(u))}{2} .
\end{aligned}
$$

This identity implies the estimate

$$
\Phi(u)+b_{1} A_{0} G_{\beta}(\Phi(u)) \leqslant B_{0}+B_{1}+b_{0} A_{0} ;
$$

here we have employed the left inequality in (9). The inequality $G_{\beta}(\Phi(u)) \leqslant \Phi(u)$ holds. This implies

$$
\left(1+b_{1} A_{0}\right) G_{\beta}(\Phi(u)) \leqslant B_{0}+B_{1}+b_{0} A_{0},
$$

or

$$
G_{\beta}(\Phi(u)) \leqslant B_{0}+B_{1}
$$

Then the identity $G_{\beta}(\Phi(u))=\Phi(u)$ holds. It means that the solution $u(x, t)$ of boundary value problem (7), (2), (3) solves the equation

$$
u_{t t}-\Delta u+\left[b_{0}-b_{1} \Phi(u)\right] u=0 .
$$

We denote $c=b_{0}-b_{1} \Phi(u)$. Then the function $u(x, t)$ and the number $c$ are related by equation (1) in the cylinder $Q$, the function $u(x, t)$ satisfies the required belongings, conditions (2) and (3) are satisfied and the number $c$ is positive. Let us show that the function $u(x, t)$ satisfies condition (4).

We denote

$$
\alpha=\int_{\Omega} u^{2}(x, T) d x .
$$

The identities

$$
c\left(A-A_{0}\right)=b_{0}-b_{1} \Phi(u), \quad c\left(\alpha-A_{0}\right)=b_{0}-b_{1} \Phi(u)
$$

hold true. This implies

$$
c(\alpha-A)=0 .
$$

We denote by $U_{0}(x, t)$ the solution to boundary value problem $(1)-(3)$ in the case $c=0$. If the identity

$$
A=\int_{\Omega} U_{0}^{2}(x, T) d x
$$

holds, then the pair $\left\{U_{0}(x, t), 0\right\}$ solves Inverse problem I and this implies immediately condition (4). If identity (18) does not hold, then the number $c$ is non-zero and thus, (17) implies $\alpha=A$. And this means that the solution $u(x, t)$ to boundary value problem $(7),(2),(3)$ satisfies all required belongings and conditions (2)-(4) hold. Hence, the pair $\left\{u(x, t), b_{0}-b_{1} \Phi(u)\right\}$ is the sought solution to Inverse problem I. The proof is complete. 
While studying the solvability of Inverse problem I with a nonzero function $f(x, t)$, an important role is played by the inequality $\Phi(u) \geqslant 0$ for solutions $u(x, t)$ to boundary value problem (7), (2). (3). We provide a simple statement giving sufficient conditions ensuring this inequality.

Let $\psi(x)$ be a function in the space $\dot{W}_{2}^{1}(\Omega)$. The identity

$$
\int_{\Omega} \psi^{2}(x) d x \leqslant m_{0} \sum_{i=1}^{n} \int_{\Omega} \psi_{x_{i}}^{2}(x) d x
$$

holds true with some number $m_{0}$ determined just by the domain $\Omega$.

We let

$$
N_{1}=\left[m_{0} N_{0}\left(e^{T}-1\right)\right]^{\frac{1}{2}}, \quad N_{2}=\left(T^{2} N_{0} e^{T}+2 T A_{0}\right)^{\frac{1}{2}},
$$

where the number $N_{0}$ was determined in the proof of apriori estimates (10) and (11) for solutions of boundary value problems $\left(7_{\varepsilon, w}\right),(2),(3)$.

Proposition 1. Let the assumptions of Theorem 1 be satisfied as well as the condition

$$
2\left(\int_{Q} f_{t}^{2} d x d t\right)^{\frac{1}{2}} \min \left(N_{1}, N_{2}\right) \leqslant 2 \int_{\Omega} f(x, 0) u_{0}(x) d x-m_{0} \int_{\Omega} f^{2}(x, T) d x .
$$

Then for the solutions $u(x, t)$ of boundary value problem (7), (2), (3) the inequality

$$
\Phi(u) \geqslant 0
$$

holds true.

Proof. We first of all observe that there exists a solution $u(x, t)$ of boundary value problem $(7)$, (2), (3) in the class mentioned in Theorem 1. For the sake of brevity we denote

$$
I(u)=\int_{\Omega} u_{t}^{2}(x, T) d x+\sum_{i=1}^{n} \int_{\Omega} u_{x_{i}}^{2}(x, T) d x
$$

The identity

$$
\Phi(u)=I(u)+2 \int_{\Omega} f(x, 0) u_{0}(x) d x-2 \int_{\Omega} f(x, T) u(x, T) d x+2 \int_{Q} f_{t} u d x d t
$$


holds true. The inequalities

$$
\begin{aligned}
& 2\left|\int_{\Omega} f(x, T) u(x, T) d x\right| \leqslant \delta^{2} \int_{\Omega} u^{2}(x, T) d x+\frac{1}{\delta^{2}} \int_{\Omega} f^{2}(x, T) d x \\
& \leqslant \delta^{2} m_{0} \sum_{i=1}^{n} \int_{\Omega} u_{x_{i}}^{2}(x, T) d x+\frac{1}{\delta^{2}} \int_{\Omega} f^{2}(x, T) d x \\
& \leqslant \delta^{2} m_{0} I+\frac{1}{\delta^{2}} \int_{\Omega} f^{2}(x, T) d x \\
& 2\left|\int_{Q} f_{t} u d x d t\right| \leqslant 2\left(\int_{Q} f_{t}^{2} d x d t\right)^{\frac{1}{2}}\left(\int_{Q} u^{2} d x d t\right)^{\frac{1}{2}} \\
& \leqslant 2\left(\int_{Q} f_{t}^{2} d x d t\right)^{\frac{1}{2}}\left(m_{0} \sum_{i=1}^{n} \int_{Q} u_{x_{i}}^{2} d x d t\right)^{\frac{1}{2}} \leqslant 2 N_{1}\left(\int_{Q} f_{t}^{2} d x d t\right)^{\frac{1}{2}}, \\
& 2\left|\int_{Q} f_{t} u d x d t\right| \leqslant 2\left(\int_{Q} f_{t}^{2} d x d t\right)^{\frac{1}{2}}\left(\int_{Q} u^{2} d x d t\right)^{\frac{1}{2}} \\
& \leqslant 2\left(\int_{Q} f_{t}^{2} d x d t\right)^{\frac{1}{2}}\left[T^{2} \int_{Q} u_{t}^{2} d x d t+2 A_{0} T\right]^{\frac{1}{2}} \leqslant 2 N_{2}\left(\int_{Q} f_{t}^{2} d x d t\right)^{\frac{1}{2}} .
\end{aligned}
$$

are valid.

In inequality (23) we fix a number $\delta$ so that $\delta^{2} m_{0}=1$. Then representation (22), inequality (23)-(25) and condition (20) imply inequality (21). The proof is complete.

Theorem 3. Let the functions $f(x, t), u_{0}(x)$ and $u_{1}(x)$ be such that

$$
f(x, t) \in L_{2}(Q), \quad f_{t} \in L_{2}(Q), \quad u_{0}(x) \in W_{2}^{2}(\Omega) \cap \stackrel{\circ}{W}_{2}^{1}(\Omega), \quad u_{1}(x) \in \stackrel{\circ}{W}_{2}^{1}(\Omega),
$$

and conditions (6) and (20) be satisfied. Then Inverse problem I has a solution $\{u(x, t), c\}$ satisfying

$$
\begin{aligned}
& u(x, t) \in L_{\infty}\left(0, T ; W_{2}^{2}(\Omega) \cap \stackrel{\circ}{W}_{2}^{1}(\Omega)\right), \quad u_{t}(x, t) \in L_{\infty}\left(0, T ; \stackrel{\circ}{2}_{2}^{1}(\Omega)\right), \\
& u_{t t}(x, t) \in L_{\infty}\left(0, T ; L_{2}(\Omega)\right), \quad c \geqslant 0 .
\end{aligned}
$$

As for Theorem 2, the proof of this theorem is based on proving the estimate

$$
G_{\beta}(\Phi(u)) \leqslant B_{0}+B_{1}
$$

at that, one should use inequality $\Phi(u) \geqslant 0$. We just mention that instead of the function $U_{0}(x, t)$ we should use the function $U_{1}(x, t)$ solving initial boundary value problem (1)-(3) in the case $c=0$.

\section{SOlVABILITY OF INVERSE PROBLEM II}

The study of the solvability of Inverse problem II is made similar to that for Inverse problem I. First we establish the solvability of the auxiliary problem (7), (2), (5) and this can be done similar to the proof of the solvability of problem $(7),(2),(3)$. Then we prove the solvability of Inverse problem II in the case $f(x, t) \equiv 0$ that can be done similar to the proof of Theorem 2. 
At the next step we prove the inequality $\Phi(u) \geqslant 0$ for the solutions $u(x, t)$ of boundary value problem (7), (2), (5). Finally, at the last step we prove the solvability of Inverse problem II for a nonzero function $f(x, t)$. The differences between this scheme and that for Inverse problem I are just in the conditions ensuring $\Phi(u) \geqslant 0$ for the solutions to boundary value problem $(7)$, $(2),(5)$. This is why we provide completely an appropriate statement.

We let

$$
N_{3}=\left(2 T N_{0}\left(e^{T}-1\right)+2 A_{0}\right)^{\frac{1}{2}} .
$$

Proposition 2. Let the functions $f(x, t), u_{0}(x)$ and $u_{1}(x)$ be such that

$$
f(x, t) \in L_{2}(Q), \quad f_{t}(x, t) \in L_{2}(Q), \quad u_{0}(x) \in W_{2}^{2}(\Omega), \quad \frac{\partial u_{0}(x)}{\partial \nu}=0
$$

as $x \in \Gamma, u_{1}(x) \in W_{2}^{1}(\Omega)$. Moreover, assume that conditions (6) are satisfied as well as the condition

$$
N_{3}\left(\int_{\Omega} f^{2}(x, T) d x\right)^{\frac{1}{2}}+N_{2}\left(\int_{Q} f_{t}^{2} d x d t\right)^{\frac{1}{2}} \leqslant \int_{\Omega} f(x, 0) u_{0}(x) d x .
$$

Then for the solutions $u(x, t)$ of boundary value problem (7), (2), (5) inequality (21) holds true.

Proof. Under the belongings for the functions $f(x, t), u_{0}(x)$ and $u_{1}(x)$ and condition (6) boundary value problem (7), (2), (5) has a solution $u(x, t)$ such that $u(x, t) \in L_{\infty}\left(0, T ; W_{2}^{2}(\Omega)\right)$, $u_{t}(x, t) \in L_{\infty}\left(0, T ; W_{2}^{1}(\Omega)\right), u_{t t}(x, t) \in L_{\infty}\left(0, T ; L_{2}(\Omega)\right)$; as it has been said above, the appropriate theorem can be proved exactly in the same way as Theorem 1. Function $\Phi(u)$ satisfies representation $(22)$, for the function $u(x, t)$ the inequalities

$$
\begin{aligned}
& 2\left|\int_{\Omega} f(x, T) u(x, T) d x\right| \leqslant 2\left(\int_{\Omega} f^{2}(x, T) d x\right)^{\frac{1}{2}}\left(\int_{\Omega} u^{2}(x, T) d x\right)^{\frac{1}{2}} \\
& \leqslant 2\left(\int_{\Omega} f^{2}(x, T) d x\right)^{\frac{1}{2}}\left[2 T \int_{Q} u_{t}^{2} d x d t+2 A_{0}\right]^{\frac{1}{2}} \leqslant 2 N_{3}\left(\int_{\Omega} f^{2}(x, T) d x\right)^{\frac{1}{2}} .
\end{aligned}
$$

hold true. Employing (27), (25), condition (26) and representation (22), we obtain inequality (21) for the function $u(x, t)$. The proof is complete.

In conclusion we formulate the solvability theorem for Inverse problem II.

Theorem 4. Let the functions $f(x, t), u_{0}(x)$ and $u_{1}(x)$ be such that

$$
f(x, t) \equiv 0, \quad u_{0}(x) \in W_{2}^{2}(\Omega), \quad \frac{\partial u_{0}(x)}{\partial \nu}=0 \quad \text { as } \quad x \in \Gamma, \quad u_{1}(x) \in W_{2}^{1}(\Omega),
$$

and condition (6) be satisfied. Then Inverse problem II has a solution $\{u(x, t), c\}$ such that

$$
\begin{array}{ll}
u(x, t) \in L_{\infty}\left(0, T ; W_{2}^{2}(\Omega)\right), & u_{t}(x, t) \in L_{\infty}\left(0, T ; W_{2}^{1}(\Omega)\right), \\
u_{t t}(x, t) \in L_{\infty}\left(0, T ; L_{2}(\Omega)\right), & c \geqslant 0 .
\end{array}
$$

Theorem 5. Let the functions $f(x, t), u_{0}(x)$ and $u_{1}(x)$ be such that

$$
\begin{aligned}
& f(x, t) \in L_{2}(Q), \quad f_{t}(x, t) \in L_{2}(Q), \quad u_{0}(x) \in W_{2}^{2}(\Omega), \\
& \frac{\partial u_{0}(x)}{\partial \nu}=0 \quad \text { as } \quad x \in \Gamma, \quad u_{1}(x) \in W_{2}^{1}(\Omega),
\end{aligned}
$$


and conditions (6) and (26) be satisfied. Then Inverse problem II has a solution $\{u(x, t), c\}$ such that

$$
\begin{array}{ll}
u(x, t) \in L_{\infty}\left(0, T ; W_{2}^{2}(\Omega)\right), & u_{t}(x, t) \in L_{\infty}\left(0, T ; W_{2}^{1}(\Omega)\right), \\
u_{t t}(x, t) \in L_{\infty}\left(0, T ; L_{2}(\Omega)\right), & c \geqslant 0 .
\end{array}
$$

The proofs of these theorems are obvious.

\section{Comments And AdDitions}

1. The technique used in the present work can be employed in many other situations. For instance, by means of this technique one can study the solvability of inverse problems with overdetermination condition (4), the unknown solution $u(x, t)$ and the unknown constant coefficient $c$

a) for Barenblatt-Zheltova-Kochina equation

34]

$$
u_{t}-a \Delta u-b \Delta u_{t}+c u=f(x, t)
$$

$(a>0, b>0)$

b) for pseudo-hyperbolic equations

$$
u_{t t}-a \Delta u-b \Delta u_{t}+c u=f(x, t)
$$

$(a>0, b>0)$

c) for non-stationary high order equations

$$
u_{t t}+(-1)^{m} \Delta^{m} u+c u=f(x, t)
$$

$m>0$ is integer.

Together with inverse problems for equations (28)-(30) with constants coefficients, it is easy to study the inverse problems similar to the studied above for some non-stationary equations with variable coefficients. For instance, employing the technique of the present work, it is easy to study the solvability of the inverse problem with conditions (2)-(4) for the equations

$$
u_{t t}-\sum_{i, j=1}^{n} \frac{\partial}{\partial x_{i}}\left(a^{i j}(x, t) u_{x_{j}}\right)+b(x, t) u_{t}+c u=f(x, t)
$$

under the conditions

$$
\begin{aligned}
& a^{i j}(x, t) \in C^{2}(\bar{Q}), \quad a^{i j}(x, t)=a^{j i}(x, t), \quad i, j=1, \ldots, n, \quad(x, t) \in \bar{Q} ; \\
& \sum_{i, j=1}^{n} a^{i j}(x, t) \xi_{i} \xi_{j} \geqslant a_{0}|\xi|^{2}, \quad a_{0}>0, \quad(x, t) \in \bar{Q}, \quad \xi \in \mathbb{R}^{n} ; \\
& \sum_{i, j=1}^{n} a_{t}^{i j}(x, t) \xi_{i} \xi_{j} \leqslant 0, \quad(x, t) \in \bar{Q}, \quad \xi \in \mathbb{R}^{n} ; \\
& b(x, t) \in C(\bar{Q}), \quad b(x, t) \geqslant b_{0}>0, \quad(x, t) \in \bar{Q} .
\end{aligned}
$$

It is easy to provide other examples of equations with variable coefficients, for which Inverse problems I and II can be studied by the technique of the present work.

2. Conditions (20) and (26) are obviously satisfied for the identically vanishing function $f(x, t)$, at that, the function $u_{0}(x)$ can be both zero or non-zero. If the function $f(x, t)$ is not identically zero, then the function $u_{0}(x)$ is not identically zero as well. Moreover, the number

$$
\int_{\Omega} f(x, 0) u_{0}(x) d x
$$


should be positive. To satisfy conditions (20) or (26), it is sufficient, for instance, if the number $T$ is small enough.

3. In the present work we do not study the uniqueness of solutions to Inverse problems I and II.

4. The results on solvability of boundary value problems (7), (2), (3) and (7), (2), (5) can be of independent interest for the theory of "loaded" equations. We note that in equation (7), the function $\Phi(u)$ can be replaced by the function $\widetilde{\Phi}(u)$ having the form

$$
\widetilde{\Phi}(u)=\int_{\Omega} u_{t}^{2}(x, T) d x+\sum_{i=1}^{n} \int_{\Omega} u_{x_{i}}^{2}(x, T) d x-2 \int_{Q} g u_{t} d x d t
$$

with the function $g(x, t)$ such that $g(x, t) \in L_{2}(Q), g_{t}(x, t) \in L_{2}(Q)$.

\section{BIBLIOGRAPHY}

1. R. Courant, D. Hilbert. Methods of mathematical physics. V. II: Partial differential equations. New York-London: Interscience Publishers, New York (1962).

2. V.S. Vladimirov. Equations of mathematical physics. Nauka, Moscow (1976). [Pure Appl. Math. 3. Marcel Dekker, New York (1971).]

3. G.V. Alexeev. Classical models and methods of mathematical physics. Dal'nauka, Vladivostok (2011). (in Russian).

4. S.I. Kabanikhin. Inverse and ill-posed problems. Theory and applications. Sibir. knizh. izd-vo, Novosibirsk (2009). [de Gruyter, Berlin (2012).]

5. A.I. Prilepko, D.G. Orlovsky, I.A. Vasin. Methods for solving inverse problems in mathematical physics. Monograph and Textbooks in Pure and Applied Mathematics. 222. Marcel Dekker Inc, New York (2000).

6. V.G. Romanov. Stability in inverse problems. Nauchnyi mir, Moscow (2005).

7. V. Isakov. Inverse problem for partial differential equations. Springer, Cham (2017).

8. M.V. Klibanov, A. Timonov. Carleman estimates for coefficient inverse problems and numerical applications. VSP, Utrecht (2004).

9. A. Lorenzi. An introduction to identification problems via funcional analysis. VSP, Utrecht (2001).

10. A.Kh. Amirov. Solvability of inverse problems // Sibir. Matem. Zhurn. 28:6, 3-11 (1987). [Siber. Math. J. 28:6, 865-872 (1987).]

11. B.A. Bubnov. On well-posedness of boundary value and inverse problems for some classes of evolution equations. Abstract of PhD thesis, Institute of Mathematics SB AS USSR, Novosibirsk (1989). (in Russian).

12. O.A. Koltunovskii. Inverse problems for hyperbolic equations with an unknown coefficients in the case of the integral overdetermination // Matem. Zametki YaGU. 15:1, 55-74 (2008). (in Russian).

13. O.A. Koltunovskii. An inverse coefficient problem for a multidimensional hyperbolic equation in the case of integral overdetermination // Matem. Zametki YaGU. 20:2, 79-97 (2013). (in Russian).

14. M.A. Kuliev. A multidimensional inverse boundary value problem for a linear hyperbolic equation in a bounded domain // Differ. Uravn. 38:1, 98-101 (2002). [Differ. Equ. 38:1, 104-108 (2002).]

15. D. Orlovsky, S. Piskarev, R. Spigler. On approximation of inverse problems for abstract hyperbolic equations // Taiwanese J. Math. 14:33, 1145-1167 (2010).

16. I.R. Valitov, A.I. Kozhanov. Inverse problems for hyperbolic equations: the case of unknown timedependent coefficients // Vestnik NGU. Ser. Matem. Mekh. Inform. 6:1, 3-18 (2006). (in Russian).

17. R.R. Saifullova. On solvability of inverse linear problem on finding a compound right hand side in a hyperbolic equation // Vestnik YurGU. Ser. Matem. Model. Progr. 37(170):4, 93-105 (2009). (in Russian).

18. A.I. Kozhanov, R.R. Safiullova. Linear inverse problems for parabolic and hyperbolic equations // J. Inver. Ill-Posed Probl. 18:1, 1-18 (2010). 
19. S.S. Pavlov. Inverse problem on recovering of an external action in a multi-dimensional wave equiation with an integral overdetermination // Matem. Zametki YaGU. 18:1, 81-93 (2011). (in Russian).

20. V.I. Priimenko, M.P. Vishnevskii. An identification problem related to the Biot system // J. Inver. Ill-Posed Probl. 23:3, 219-230 (2015). (in Russian).

21. C. Cavaterra, A. Lorenzi, M. Yamamoto. A stability result via Carleman estimates for an inverse source problem related to a hyperbolic integro-differential equation // Comput. Appl. Math. 25:2-3, 229-250 (2006).

22. A. Lorenzi, E. Paparoni. Identifications of two unknown coefficients in an integro-differential hyperbolic equation // J. Inv. Ill-Posed Probl. 1:4, 331-348 (1993).

23. L. Seliga, M. Slodicka. An inverse source problem for a damped wave equation with memory // J. Inv. Ill-Posed Probl. 24:2, 111-122 (2015).

24. A. Lorenzi, G. Mola. Identification of a real constant in linear evolution equation in a Hilbert spaces // Inver. Probl. Imaging. 5:3, 695-714 (2011).

25. G. Mola. Identification of the diffusion coefficient in linear evolution equations in Hilbert spaces // J. Abstr. Diff. Equat. Appl. 2:1, 18-28 (2011).

26. A. Lorenzi, G. Mola. Recovering the reaction and the diffusion coefficients in a linear parabolic equation // Inver. Probl. 28:7, id 075006 (2012).

27. M.T. Dzhenaliev. To the theory of linear boundary value problems for loaded differential equations. Instit. Teor. Prikl. Matem., Almaty (1995).

28. A.M. Nakhushev. Loaded equations and their applications. Nauka, Moscow (2012). (in Russian.)

29. A.I. Kozhanov. To the theory of compound equations. Abstract of Habilitation thesis, Novosibirsk (1993). (in Russian).

30. A.I. Kozhanov Composite type equations and inverse problems. VSP, Utrecht (1999).

31. S.L. Sobolev. Some applications of functional analysis to mathematical physics. Nauka, Moscow (1988). [Transl. Math. Monog. 90. Amer. Math. Soc. Providence, RI (1991).]

32. O.A. Ladyzhenskaya, N.N. Ural'tseva. Linear and quasilinear elliptic equations. Nauka, Moscow (1973). [ Math. Sci. Engineer. 46. Academic Press, New York (1968).]

33. V.A. Trenogin. Functional analysis. Nauka, Moscow (1980). (in Russian).

34. G.I. Barenblatt, Yu.P. Zheltov, I.N. Kochina. Basic concepts in the theory of seepage of homogeneous liquids in fissured rocks (strata) // Prikl. Mat. Mekh. 24:5, 852-864 (1960). [J. Appl. Math. Mech. 24:5, 1286-1303 (1961).]

Alexander Ivanovich Kozhanov,

Sobolev Institute of Mathematics, SB RAS,

Acad. Koptyug av. 4,

630090, Novosibirsk, Russia

E-mail: Kozhanov@math.nsc.ru

Regina Rafailovna Safiullova,

Sobolev Institute of Mathematics, SB RAS,

Acad. Koptyug av. 4,

630090, Novosibirsk, Russia

E-mail: regina-saf@yandex.ru 